\title{
FADIGA NO TRABALHO: COMO O PSICÓLOGO PODE ATUAR?
}

\author{
Juliana Roman dos Santos Oliveira* \\ Magna Gabriella Viganó \\ Maria Cristina Frollini Lunardelli \\ Luiz Carlos Canêo" \\ Edward Goulart Júnior
}

\begin{abstract}
RESUMO. As más condições laborais e a forma de organização do trabalho dentro do sistema capitalista frequentemente têm se mostrado nocivas para o trabalhador. Além disso, a chamada síndrome da fadiga crônica é um dos males que acomete a classe trabalhadora. Por meio de revisão teórica buscou-se caracterizar a síndrome, suas possíveis causas e consequências e o papel do psicólogo nas organizações. A fadiga pode ser compreendida como um esgotamento físico e mental, culminando em consequências para a saúde do trabalhador. Excesso de trabalho, pressão por produção, turnos alternados, riscos físicos e falta de autonomia são alguns fatores apontados como prováveis desencadeadores da síndrome, e as consequências encontradas vão desde baixo rendimento e maior risco de acidentes de trabalho até dificuldade para relaxar em momentos de lazer. É papel do psicólogo do trabalho e organizacional realizar ações remediadoras e preventivas que impeçam ou dificultem o aparecimento da fadiga no trabalho.
\end{abstract}

Palavras-chave: Fadiga no trabalho; psicologia organizacional; saúde do trabalhador.

\section{FATIGUE AT WORK: HOW CAN THE PSYCHOLOGIST ACT?}

\begin{abstract}
Poor working conditions and the organized way of work inside the capitalist system have frequently been harmful to the worker. Besides, the Chronic Fatigue Syndrome is one of many disorders that can affect the working class. Through a theoretical revision, there has been an attempt to characterize the syndrome, its possible causes and consequences, also the role of psychologist inside the organizations. The fatigue can be understood as a physical and mental exhaustion, culminating in consequences to worker's health. Overworking, pressures for production, switched shifts, physical hazards and lack of autonomy are some factors mentioned as likely of triggers of the syndrome, and the verified consequences range from little productivity and major risk of accidents to difficulties in enjoying free time. It is the job of the work and organizational psychologist to perform preventive and remedial actions which lessen the emergence of fatigue at work.
\end{abstract}

Key words: Fatigue at work; organizational psychology; worker's health.

\section{FATIGA EN EL TRABAJO: ¿CÓMO EL PSICÓLOGO PUEDE ACTUAR?}

RESUMEN. Las más condiciones de trabajo y la forma de organización en el sistema capitalista se mostra mala para el trabajador. También, el Síndrome de la sobrecarga crónica, es uno de los males que rondan los trabajadores hoy. Por el medio de la revisión teórica, se intentó caracterizarlo, sus posibles causas, consecuencias y la función del psicólogo en las organizaciones. La sobrecarga puede ser comprendida como un agotamiento físico y mental, acarreando en consecuencias para su salud. El exceso de trabajo, producción, turnos alternos, riesgos físicos y falta de autonomía son factores apuntados como probables desencadenadores del síndrome y sus consecuencias son bajos réditos, mayores riesgos de accidentes de trabajo y dificultad para relajar en los ratos de recreación. Es la función del psicólogo del trabajo y organizacional realizar acciones remediables y preventivas que impidan el aparecimiento de la sobrecarga en el trabajo..

Palabras-clave: Fatiga en el trabajo; Psicología organizacional; Salud del trabajador.

Psicóloga da Universidade Estadual Paulista-UNESP-Bauru.

\# Doutora em Educação e docente de Psicologia da Universidade Estadual Paulista-UNESP-Bauru.

II Doutor em Educação e docente de Psicologia da Universidade Estadual Paulista-UNESP-Bauru. 


\section{CARACTERIZAÇÃO DA SÍNDROME}

Desde a Revolução Industrial, no início do século XVIII, a forma de organização do trabalho tem suscitado inúmeras reclamações por parte dos trabalhadores. As queixas vão desde ambientes insalubres de trabalho, exposição a produtos tóxicos, falta de equipamentos de segurança e condições inadequadas de trabalho (má luminosidade, barulho, ferramentas obsoletas, etc.) até longas jornadas de trabalho, baixos salários, autoritarismo, ameaça de desemprego, pressão por produção, falta de autonomia do empregado e outros. Todos esses fatores têm consequência direta na vida dos trabalhadores no que diz respeito à sua saúde física e mental.

O psicólogo inserido em organizações de trabalho tem o dever de zelar pela saúde do trabalhador, o que se dá não somente com ações remediadoras, mas sobretudo por meio de práticas que previnam a instalação e aparecimento de transtornos mentais. Muitas são as complicações que podem advir da relação do trabalhador com sua ocupação, como depressão, transtornos afetivos, alteração do ciclo vigília-sono, neurastenia, burnout e outras.

$\mathrm{O}$ interesse pelas síndromes de cansaço em suas diversas apresentações e denominações, como astenia (fadiga prévia ao esforço) ou simplesmente fadiga (impossibilidade de manter esforço), é antiga, pois os pacientes acometidos por estas síndromes acabam se tornando grandes usuários dos serviços públicos de saúde (Bertolín \& Calvo, 1997).

Historicamente, a fadiga recebeu diversas definições e denominações, como neurastenia (1869), psicastenia (1903), brucelose crônica (1947), fadiga vital (1966), entre outras, e foi apenas em 1986, nos Estados Unidos, que foi oficialmente reconhecida e nomeada como "síndrome da fadiga crônica" (SFC).

Apesar de esta última ser a denominação mais recente, as outras não são menos legítimas que ela, pois, segundo Kleiman (1998), as formas e funções dos transtornos mentais não estão no mundo natural, mas resultam da interação entre estruturas sociais e experiências pessoais. Neste sentido, o diagnóstico da SFC, segundo o autor, resulta da interação entre o conceito e a experiência num determinado contexto cultural.

Segundo Fukuda et al. (1994), a importância maior não consiste em denominações ou categorizações da síndrome - de modo que as interpretações dos sintomas possam tornar-se reducionistas - , mas sim, em considerar o aspecto dimensional da fadiga, e não apenas o categórico. Por essa razão, atualmente persiste a necessidade de revisão dos critérios diagnósticos, avaliação diagnóstica e utilização de uma abordagem integrativa e compreensiva desse transtorno.

A fadiga é considerada por diversos autores como um esgotamento físico e mental grave e crônico que difere do cansaço e da falta de motivação por não ser atribuída a exercício físico ou a uma enfermidade diagnosticável. É um fenômeno preocupante e de difícil conceituação, interpretação e aferição, porque acaba por nomear um estado global resultante do desequilíbrio interno devido ao sistema de relações do organismo, no qual muitas vezes a alteração de um sistema afeta os demais.

Entende-se que a fadiga pode atingir indivíduos de todas as faixas etárias no desenvolvimento de qualquer tipo de atividade realizada por um período de tempo. Além de ser um fenômeno que causa mal-estar, ela provoca alterações no estado psicossomático, podendo ser encarada como resultante de esforço físico e/ou mental associado às condições ambientais e psicológicas, individuais e de trabalho (Marziale \& Rozestraten, 1995).

De acordo com a classificação proposta por Fukuda et al. (1994) no artigo The chronic fatigue syndrome: a comprehensive approach to its definition and study, um dos mais citados na caracterização da SFC, essa síndrome pode ser diagnosticada de acordo com os seguintes critérios: (1) presença de fadiga crônica relevante, inexplicável, persistente ou recorrente, clinicamente avaliada de início recente ou definitivo [não ocorreu durante a vida], não resultante de exercício em curso, que não pode ser aliviada pelo descanso e que reduz substancialmente os níveis precedentes de atividades ocupacionais, educacionais, sociais ou pessoais; (2) a ocorrência simultânea de quatro ou mais dos seguintes sintomas, que devem persistir ou recorrer durante seis meses consecutivos ou mais e não devem ter ocorrido antes do início da fadiga: prejuízo na memória ou concentração, a curto prazo, suficiente para causar a redução substancial nos níveis precedentes de atividades ocupacionais, educacionais, sociais ou pessoais; garganta inflamada; linfonodos cervicais ou axilares doloridos; dores musculares, dores nas articulações sem inchaço ou vermelhidão; dores de cabeça de um novo tipo, padrão ou severidade; sono não reparador; e fraqueza pósexercício que dure mais de 24 horas.

$\mathrm{Na}$ atual versão da classificação internacional de doenças (CID-10), a SFC está incluída na entidade nosológica neurastenia, dentro da família classificatória dos transtornos neuróticos, mas não está descrita no Manual de Diagnóstico e Estatística das Perturbações Mentais (DSM-IV). Na CID-10, as 
diretrizes para um diagnóstico definitivo apontam para as seguintes queixas: fadiga aumentada após esforço mental; fraqueza e exaustão após esforço mínimo; sintomas autonômicos ou depressivos não classificados em outros diagnósticos da CID-10 e duas ou mais das queixas de dores musculares, tonturas, cefaleias, perturbação do sono, irritabilidade e dispepsia.

Ainda, em relação ao diagnóstico da síndrome, Cho (2007) refere-se ao questionário de fadiga de Chalder (CFQ), o qual tem como objetivo tanto medir a gravidade das fadigas física e mental quanto auxiliar na avaliação de pacientes portadores da SFC. Este instrumento, de origem inglesa, foi adaptado por Cho para a realidade brasileira, mostrando-se válido e confiável nos referidos diagnósticos.

Em 2009 o autor acima citado realizou um estudo utilizando-se do CFQ, com o objetivo de diagnosticar a SFC em unidades de atendimento básico de saúde em Londres e no Brasil (São Paulo). O autor conclui que a predominância da SFC foi similar nos dois países, apesar das diferenças socioculturais e econômicas entre ambos.

Diante do exposto, considera-se a SFC relevante no contexto organizacional, pois está relacionada às condições de trabalho e se reflete no desempenho do empregado, além de afetar negativamente sua saúde física e mental. Os objetivos deste trabalho consistem em fazer uma revisão teórica com a apresentação de diversas pesquisas que apontam a relação da SFC com o ambiente e as condições de trabalho e levantar algumas formas de atuação do psicólogo em prevenção e intervenção que auxiliem no manejo da síndrome.

\section{FATORES NO AMBIENTE DE TRABALHO QUE PODEM PROPICIAR O APARECIMENTO DA FADIGA}

O estudo de Glina, Rocha, Batista e Mendonça (2001) apresenta um caso de um trabalhador de linha de produção diagnosticado com neurastenia, de acordo com o CID-10, cuja causa comprova-se estar relacionada com as condições de trabalho. Entre os fatores apontados como geradores do sintoma estão o excesso de trabalho e a pressão por produção, "a existência de ritmo intenso, falta de autonomia, falta de reconhecimento em relação ao desempenho, a automação gerando desemprego e modificando o processo de trabalho sem a participação dos trabalhadores" (p. 611).

Outro estudo, conduzido por Marziale e Rozestraten (1995), aponta a presença de fadiga mental nos trabalhadores de enfermagem que atuam em instituições hospitalares com esquema de trabalho em turnos alternados. Nesse estudo, do qual participaram doze enfermeiras de três diferentes unidades de internação, foram analisadas cinco jornadas consecutivas, nos turnos da manhã, tarde e noite. Os resultados indicam que a alternância entre turnos é prejudicial à saúde e à vida social, familiar e profissional dessas enfermeiras, as quais revelaram insatisfação com o esquema de trabalho e apresentaram sintomas de fadiga mental. A incidência desses sinais foi maior no turno da noite, em seguida no turno da manhã e, em terceiro lugar, no da tarde. Isso indica que a troca de turnos é um fator importante para o surgimento da fadiga no trabalho, apontando que o trabalho à noite tende a ser mais prejudicial à saúde do trabalhador do que os demais períodos.

Não obstante, Metzner e Fischer (2001), em estudo realizado com trabalhadores de uma indústria têxtil a fim de analisar a relação entre fadiga e turno de trabalho de doze horas com semana reduzida, obtiveram resultados de menor grau de fadiga em trabalhadores do período noturno do que nos de período diurno. Considerando que os fatores que influenciaram na percepção da fadiga se associam a estilos de vida dos funcionários (como prática de exercícios físicos ou outro fator protetor) e à incapacidade de manter o sono, os autores ainda ressaltam que os trabalhadores participantes desse estudo desenvolveram estratégias de enfrentamento eficazes, enquanto aqueles que não se adaptaram ao turno noturno não participaram do grupo.

Outros autores (Oliniski, Rothbarth, Ulbrich \& Felibino, 2005) apontam que o ambiente de trabalho em saúde é naturalmente gerador de fadiga e mobilizador de emoções em seus trabalhadores. As atividades em turnos alternados, jornada de trabalho extensa, risco químico, biológico e físico, necessidade de atenção constante, elevado nível de complexidade para o desenvolvimento das ações, a própria natureza do trabalho e o fato de lidar com o sofrimento, dor e morte são fatores agressores à saúde e ao bem-estar dos profissionais e podem deixá-los suscetíveis à fadiga decorrente do trabalho.

No estudo de Hernández-Penã, Kageyama, Coria, Hernández e Harlow (1999) com trabalhadoras ambulantes, a fadiga ocupacional foi associada a condições de trabalho como: longa jornada, posturas corporais mantidas por tempo prolongado, esforço físico, estresse e alguns elementos da exposição ambiental. Apontam ainda que as longas jornadas e a ausência de dias de repouso acabam se tornando 
características do trabalho informal, pois este não tem respaldo da lei.

Rocha e Debert-Ribeiro (2001) encontraram a sobrecarga de trabalho devido a prazos curtos, o alto grau de responsabilidade, a exigência mental do trabalho e a complexidade da tarefa como fatores de incômodo para os trabalhadores de análise de sistemas, com repercussões na saúde, sendo a fadiga apontada em grande porcentagem das mulheres.

Quevedo, Palma e Quintero (2005) revelaram que os trabalhadores de uma cervejaria venezuelana apresentaram maiores índices de fadiga quando expostos a excesso de ruídos e baixa iluminação, o que indica que fatores ambientais podem ser coadjuvantes no desenvolvimento dessa síndrome.

A síndrome da fadiga pode surgir, ocasionalmente, em trabalhadores expostos a alguns produtos químicos, como tolueno, tricloroetileno, tetracloroetileno, tricloetano, manganês, mercúrio, sulfeto de carbono e outros solventes orgânicos neurotóxicos (Ministério da Saúde do Brasil, 1999). Indústrias que empregam estes produtos químicos no seu processo de produção devem tomar todas as medidas de segurança do trabalhador para evitar que estes se contaminem com tais compostos, prevenindo o desenvolvimento da SFC no seu quadro de funcionários.

Os estudos apresentados indicam que a estrutura atual do trabalho (longa jornada, pressão por produção, falta de autonomia, pouco tempo para descanso e férias) e condições como alternância de turnos, riscos físicos, complexidade da atividade, postura durante a execução, entre outras, favorecem o aparecimento da fadiga, a qual pode manifestar-se em trabalhadores de diferentes tipos de atividade desde os operários até os profissionais da saúde. Do mesmo modo, também as formas de manifestação desta vão se dar em diferentes níveis, a depender da relação do indivíduo com a atividade nociva.

\section{CONSEQUÊNCIAS DA FADIGA PARA O TRABALHO E PARA A SAÚDE DO TRABALHADOR}

O trabalho é uma atividade inerente ao indivíduo enquanto ser social. Como, o homem passa grande parte de sua vida no ambiente laboral, ele está sujeito a diversos tipos de intercorrência que poderão repercutir negativa ou positivamente sobre sua saúde física e mental. O trabalho, para Dejours, Dessors e Desriaux (1993), é um fator de equilíbrio, de satisfação e de desenvolvimento pessoal, mas pode também constituir-se em um fator de infelicidade e de envelhecimento e causador de doenças. O resultado disso na saúde do trabalhador, seja este prazeroso ou penoso, vai depender de ele encontrar uma atividade equilibrada - fonte de realização profissional - ou um trabalho fatigante - fonte de insatisfação, desmotivação, estresse e de doenças ocupacionais.

Como consequência de fatores intrínsecos e extrínsecos ao trabalhador, a fadiga traz para o ambiente organizacional diversas implicações, tanto para a saúde do trabalhador quanto para a empresa, que precisa arcar com os prejuízos de um ou mais funcionários portadores da síndrome.

Entre as diversas consequências no ambiente de trabalho, a fadiga pode causar baixo rendimento, altos índices de absenteísmo, risco elevado de se envolver em acidentes de trabalho ou cometer erros na atividade desenvolvida, desenvolvimento de lesões de esforço, entre outros. Soma-se a isso o fato que em determinadas ocupações, como a de enfermeiros e motoristas, erros e acidentes de trabalho são inadmissíveis, pois envolvem riscos ao trabalhador e à vida de terceiros.

Hernández-Penã et al. (1999) buscaram analisar a relação entre as condições de trabalho e a saúde reprodutiva na mulher, estudando os efeitos negativos na reprodução de acordo com o tipo de atividade - no caso, o trabalho ambulante. Encontrou-se como repercussão mais frequente o baixo peso dos bebês ao nascer (a fadiga, nesse caso, revelou-se um fator determinante), os abortos e as malformações congênitas nos fetos.

Em estudo envolvendo analistas de sistemas, constatou-se que, entre as mulheres, houve uma incidência maior de fadiga física e mental. (Rocha \& Debert-Ribeiro, 2001). Além disso, a maioria dos homens e mulheres entrevistados sofre fadiga após o trabalho, havendo interferência também na escolha do lazer. A fadiga ocupacional, cuja origem está no local de trabalho, acaba interferindo na vida cotidiana do trabalhador, impedindo-o inclusive de desfrutar dos momentos de lazer.

\section{FADIGA NO TRABALHO E A ATUAÇÃO DO PSICÓLOGO ORGANIZACIONAL}

Conforme já exposto, a relação entre trabalho e saúde é bastante complexa e precisa de uma ampla gama de olhares, de diferentes profissionais, além das contribuições que o psicólogo pode realizar. Não temos a pretensão de esgotar as possibilidades de reflexão sobre o tema tratado, mas, sim, apresentar algumas considerações a respeito. 
Inicialmente é importante compreender a fadiga no trabalho como resultado de um processo social (sem desconsiderar a dimensão biológica que é, também, histórica e social), considerando-se que importantes transformações sociais vêm ocorrendo nos últimos tempos, as quais, no tocante ao mundo do trabalho, vêm acarretando profundas transformações na organização do trabalho e na vida do trabalhador.

Cumpre, não obstante, ressaltar que não é o trabalho em si que é nocivo ou representa perigo, mas sim, a forma como ele é organizado. Há que se pensar e investir esforços para que o trabalho possa ser ocasião e motivo de desenvolvimento de potencialidades individuais e coletivas, de satisfação e de crescimento, além de fonte de bem-estar físico e psíquico.

Uma boa iniciativa nessa direção poderia começar com o resgate e valorização do saber acumulado pelo grupo de trabalhadores, libertando-os dos rígidos controles impostos pelas máquinas e pelas formas perversas de organização do trabalho que o excluem do pensar sobre o seu fazer.

Nesse contexto, cabe ao psicólogo e demais profissionais da organização envolvidos com essa questão avaliar os riscos a que os trabalhadores estão expostos. O psicólogo, em particular, pode realizar tanto trabalhos preventivos à fadiga no trabalho quanto ações remediadoras, nos casos da síndrome já instaurada. Segundo Cury, Daré, Goulart, Canêo e Lunardelli (2006), ele deve buscar parceria com outros profissionais da organização, a fim de, juntos, refletirem sobre o problema da fadiga profissional e delimitarem ações a curto, médio e longo prazo. É importante que o psicólogo busque agir em conjunto com as outras estruturas de promoção da saúde do trabalhador já presentes na organização, como o Serviço Especializado em Engenharia de Segurança e Medicina do Trabalho (SESMT) e a Comissão Interna de Prevenção de Acidentes (CIPA), a fim de se realizar um trabalho conjunto e multidisciplinar, formando equipes de trabalho.

Quanto às ações preventivas, o psicólogo pode analisar as situações de trabalho em relação ao modo de organização, ao ambiente físico, ao relacionamentos entre chefias e subordinados, entre outras, e propor, por meio de um diagnóstico organizacional, mudanças que sejam benéficas à saúde do trabalhador. Também é possível elaborar um programa preventivo em ambientes vulneráveis ou nos quais haja trabalhadores com SFC ou síndromes relacionadas, abordando os aspectos pessoais e organizacionais que podem contribuir para o desenvolvimento dessas doenças.
Ainda visando à prevenção, o psicólogo pode exercer o papel de educador dentro da organização, tanto mantendo diálogo permanente com a chefia, a fim de apontar fatores organizacionais que possam propiciar o aparecimento da fadiga e mencionar as prováveis consequências para a própria organização, quanto promovendo palestras informativas, discussões e debates sobre o tema entre os trabalhadores, a fim de que estes identifiquem o surgimento do problema e o(s) evento(s) causador(res) e sugiram modificações no ambiente de trabalho que melhorem suas condições de saúde.

Em relação às ações remediadoras, o profissional de psicologia inserido nas organizações deve orientar o trabalhador a procurar um perito em análise de doenças associadas ao trabalho ou recorrer ao Centro de Referência à Saúde do Trabalhador (CRST), onde é possível caracterizar a doença e fazer o nexo causal do transtorno com o trabalho a partir de documentos específicos, como a Comunicação de Acidente de Trabalho (CAT). Assim, o empregado poderá exigir dos órgãos públicos benefícios como o auxílio-doença, o auxílio-acidente e pedidos de afastamento, assim como ser encaminhado para serviços públicos ou conveniados de saúde mental que ofereçam tratamento e apoio psicológico.

Destarte, é dever do psicólogo buscar conhecimento sobre doenças relacionadas ao trabalho e seus sintomas (como a fadiga), a fim de permitir a identificação de trabalhadores que estejam sofrendo de transtornos mentais provenientes de sua condição de trabalho, bem como efetuar/propor as devidas mudanças no ambiente laboral. É complementar a elaboração de programas de readaptação ao empregado afastado por motivo de fadiga (ou qualquer outro transtorno relacionado ao trabalho) quando este retorna às atividades, para preveni-lo do contato com os mesmos (ou novos) fatores nocivos e assim evitar uma recaída.

\section{CONSIDERAÇÕES FINAIS}

E modo geral a SFC ainda é pouco discutida e a bibliografia a respeito desta síndrome é escassa, por isso poucas práticas que visem a atuação em todos os níveis (preventivos e remediadores) são observadas no exercício dos psicólogos no espaço institucional. O objetivo deste trabalho foi apresentar uma revisão teórica, citando diversas pesquisas que apontam a relação da SFC com o ambiente e as condições de trabalho, e levantar algumas possibilidades de atuação do psicólogo, inclusive em equipe, em prevenção e intervenção voltadas para essa síndrome. 
É imprescindível que os pesquisadores se voltem para o estudo da fadiga no trabalho, uma vez que ainda não há consenso sobre seus critérios diagnósticos e a doença nem sequer está inserida nos manuais diagnósticos de transtorno mental (DSMs), portanto também não estão disponíveis dados epidemiológicos e de distribuição populacional, nem informações sobre prognóstico e evolução.

É importante ressaltar que a SFC deve ser diagnosticada por meio de uma abordagem integrativa e compreensiva (aspecto dimensional) e que leve em conta as facetas biopsicossociais do indivíduo portador dos sintomas, o que justifica a importância da Psicologia na atuação com esta síndrome, especialmente no contexto organizacional. Esta prática do psicólogo caracteriza-se como emergente por prevenir o transtorno ou identificá-lo antes que ele se intensifique. É também uma forma de tratar o trabalhador como sujeito, um ser humano cujos direitos devem ser respeitados e cuja saúde, tanto física quanto mental, deve ser estabelecida como uma das prioridades na política da organização.

\section{REFERÊNCIAS}

Bertolín, J. M. \& Calvo, J. (1997). Síndrome de fatiga crónica: ¿Ser o no ser?. Editorial, Revista Medicina Clínica, 108(15), 577579.

Cho, H. J., Costa, E., Menezes, P. R., Chalder, T., Bhugra, D. \& Wessely, S. (2007). Cross-cultural validation of Chalder Fadigue Questionnaire in Brasilian primary care. Journal of Psychosomatic Research, 62, 301-304.

Cho, H. J., Menezes, P. R., Hotopf, M., Bhugra, D. \& Wessely, S. (2009). Comparative epidemiology of chronic fatigue syndrome in Brazilian and British care: prevalence and recognition. The British journal of psychiatry, 194, 117-122.

Cury, M., Daré, M. F., Goulart Júnior, E., Canêo, L. C. \& Lunardelli, M. C. F. (2006). Fadiga profissional: consequências indesejáveis para os indivíduos e para as organizações. Em E. Goulart Júnior, L. C. Canêo, M. C. F. Lunardelli, S. E. Sposito (Org.). Contribuições do psicólogo para a promoção da saúde, qualidade de vida do trabalhador e desenvolvimento das organizações. (Vol. 01, pp. 135-141) Bauru, SP: Joarte.
Dejours, C., Dessors, D. \& Desriaux, F. (1993). Por um trabalho, fator de equilíbrio. Revista de Administração de Empresas, 33(3), 98-104.

Fukuda, K., Straus, S., Hickie, I., Sharpe, M. C., Dobbins, J. G. \& Komaroff, A. (1994). The chronic fatigue syndrome: a comprehensive approach to its definition and study. Annals of Internal Medicine, 121(12), 953-959.

Glina, D. M. R., Rocha, L. E., Batista, M. L. \& Mendonça, M. G. V. (2001). Saúde mental e trabalho: uma reflexão sobre o nexo com o trabalho e o diagnóstico, com base na prática. Caderno de Saúde Pública, 17(3), 607-616.

Hernández-Penã, P., Kageyama, M. L., Coria, I., Hernández, B. \& Harlow, S. (1999). Condiciones de trabajo, fatiga laboral y bajo peso al nacer en vendedoras ambulantes. Salud Pública, 4l(2), 101-109.

Kleiman, A. (1998). Rethinking psychiatry: from cultural category to personal experience. New York: The Free Press.

Marziale, M. H. P. \& Rozestraten, R. J. A. (1995). Turnos alternantes: fadiga mental de enfermagem. Revista Latinoamericana de Enfermagem, 3(1), 59-78.

Metzner, R. J. \& Fischer, F. M. (2001). Fadiga e capacidade para o trabalho em turnos fixos de doze horas. Revista de Saúde Pública, 35(6), 548-553.

Ministério da Saúde do Brasil. (2001). Doenças relacionadas ao trabalho: manual de procedimentos para os serviços de saúde (Portaria $n^{\circ}$ 1.339/99). Brasília, DF: Ministério da Saúde do Brasil.

Oliniski, S. R., Rothbarth, S., Ulbrich, E. M. \& Felisbino, K. (2005). A fadiga no trabalho em saúde: revisão bibliográfica. Resumos do Congresso Brasileiro de Enfermagem, 57, Goiânia, GO. Recuperado em 25 de junho, 2008, de http://bvsms.saude.gov.br/bvs/publicacoes/57cbe/resumos/757. htm.

Quevedo, A. L., Palma, A. L. \& Quintero, M. M. (2005). Fatiga laboral y condiciones ambientales en una planta de envasado de una industria cervecera. Revista Salud de los Trabajadores, 13(1), 37-44.

Rocha, L. E. \& Debert-Ribeiro, M. (2001). Trabalho, saúde e gênero: estudo comparativo sobre analistas de sistemas. Revista de Saúde Pública, 35(6), 539-547.

Recebido em 12/12/2008 Aceito em 19/03/2010
Endereço para correspondência:
Maria Cristina Frollini Lunardelli. Universidade Estadual Paulista Júlio de Mesquita Filho, Faculdade de Ciências de Bauru, Departamento de Psicologia. Avenida Luiz Edmundo Carrijo Coube, 14-01, Vargem Limpa, CEP: 17033-360, Caixa-Postal: 473, Bauru-SP, Brasil. E-mail: mlunardelli@uol.com.br. 